\title{
EL CURSO DE ACTUALIZACIÓN PARA DOCENTES DE ESTUDIOS SOCIALES DE LA DIRECCIÓN REGIONAL DE EDUCACIÓN DE HEREDIA: UNA EXPERIENCIA DESDE EL PROYECTO DE EDUCACIÓN CONTINUA BIMODAL
}

\section{THE BIMODAL UPDATE COURSE FOR SOCIAL STUDIES TEACHERS OF THE REGIONAL EDUCATION DIRECTORATE OF HEREDIA. AN EXPERIENCE FROM THE BIMODAL CONTINUOUS EDUCATION PROJECT}

\author{
Alejandra Barquero Ruiz* \\ Roberto Granados Porras**
}

Fecha de recepción: 02/09/2019

Fecha de aceptación: 01/10/2019

Resumen: En el presente artículo se pretende desarrollar la experiencia del curso bimodal de actualización para docentes de Estudios Sociales de la Dirección Regional de Educación en Heredia, Costa Rica. El curso contó con la asistencia de 39 docentes de secundaria, quienes participaron de sesiones presenciales y virtuales durante cinco meses -20 horas presenciales y 20 horas virtuales-, donde se abordaron temáticas como: historia ambiental, revoluciones sociales en América Latina, refugiados ambientales en el siglo XXI, el islam y sociedades cristianas y musulmanas en la Edad Media y el rol geopolítico de China en el contexto de una economía globalizada. Las sesiones fueron desarrolladas por especialistas de la Escuela de Historia y de la Escuela de Relaciones Internacionales y por medio de sesiones virtuales en el Aula Virtual de la Universidad Nacional (UNA). Las temáticas del curso se seleccionaron en conjunto con la asesora de Estudios Sociales de la Dirección Regional de Educación de Heredia, quien manifestó la necesidad de actualizar al colectivo docente que impartía sétimo y décimo año de los nuevos planes de estudio aprobados en el año 2016.

Palabras claves: estudios sociales; educación continua; educación bimodal; tecnología educativa; virtualidad.

\begin{abstract}
For this article, we intend to develop the experience of the bimodal update course for Social Studies teachers of the Regional Education Directorate of Heredia. The course was attended by 39 high school teachers, who participated in face-to-face and virtual sessions for four months -20 contact hours and 20 virtual hours-, addressing topics such as: Environmental History; Social Revolutions in Latin America; Environmental refugees in the 21st century; Islam and Christian and Muslim societies in the

* Costarricense. Máster en Gerencia de Proyectos. Licenciada en la Enseñanza de los Estudios Sociales y la Educación Cívica. Docente de la Escuela de Historia de la Universidad Nacional (UNA), Costa Rica. Correo electrónico: alejandra.barquero.ruiz@una.cr

** Costarricense. Licenciado en la Enseñanza de los Estudios Sociales y la Educación Cívica. Máster en Historia Aplicada. Docente e investigador de la Escuela de Historia de la Universidad Nacional (UNA), Costa Rica. Correo electrónico: c.roberth@gmail.com
\end{abstract}


El curso de actualización para docentes de Estudios Sociales de la Dirección Regional de Educación de Heredia: una experiencia desde el Proyecto de Educación Continua Bimodal

Middle Ages; the geopolitical role of China in the context of a globalized economy. The sessions were developed by specialists from the School of History and the School of International Relations. The virtual sessions were developed through the Virtual Classroom of the National University (UNA). The subjects of the course were selected in conjunction with the Social Studies Advisor of the Regional Directorate of Education of Heredia who expressed the need to update the teachers who taught seventh and tenth year in some contents of the new study programs.

Keywords: Social Studies; Continuing Education; Bimodal Education; Educational Technology; Virtuality.

\section{Introducción}

En mayo de 2016 el Consejo Superior de Educación aprobó los nuevos Programas de Estudio para Estudios Sociales en III Ciclo y Educación Diversificada -acuerdo No 03-25-2016-. Según el Ministerio de Educación Pública (MEP), estos pretenden romper con el abordaje tradicional de la historia y la geografía, incorporando una perspectiva que va de lo local a lo global. Se intenta promover el pensamiento crítico mediante un aprendizaje orientado por preguntas, que promueva la construcción del conocimiento, respetando la diversidad y multiculturalidad. Incorpora temas de relevancia actual, como el recurso hídrico, el cambio climático y las migraciones (Presidencia de la República, 2016). Ante los retos que generó la nueva propuesta curricular, se requiere actualizar al profesorado sobre diversas temáticas y metodologías.

La Escuela de Historia posee un proyecto integrado de extensión y docencia denominado Proyecto de Educación Continua Bimodal para docentes de Estudios Sociales y Educación Cívica, cuyo objetivo básico es ofrecer actividades formativas que contribuyan con la formación y actualización de los docentes de estas áreas. Se ofertan cursos virtuales y bimodales de 40 horas de aprovechamiento y se coordinan cursos presenciales -de entre 4 y 8 horasdependiendo de la demanda de las Direcciones Regionales de Educación.

Partiendo de esta necesidad, el Proyecto de Educación Continua Bimodal coordinó con la Asesoría Regional de Estudios Sociales de la Dirección Regional de Heredia la formulación de un curso bimodal cuyo objetivo era actualizar a los docentes sobre contenidos introducidos en los nuevos programas de estudio. Se contó con la participación de 39 docentes de secundaria. El curso se propuso a esta Dirección Regional de Educación debido a las facilidades brindadas por esta en cuanto a la convocatoria de los docentes y a la logística del curso. Los contenidos fueron definidos en conjunto con la asesora regional de Estudios Sociales MSc. Jenny Contrera Briceño.

El presente trabajo tiene como objetivo compartir la experiencia generada a partir de la implementación del curso de actualización para docentes de Estudios Sociales de la Dirección Regional de Educación de Heredia, como manera de contribuir a mejorar el proceso de enseñanza y aprendizaje de la Historia. 


\section{Principios teórico-metodológicos}

Para iniciar, se debe aclarar la concepción de los Estudios Sociales y la Educación Cívica. De acuerdo con la propuesta del Ministerio de Educación Pública (2016):

"Los Estudios Sociales no refieren expliícitamente a una ciencia o disciplina, en el sentido de que no poseen un objeto de estudio, teorías y métodos particulares en comparación con otras áreas del conocimiento (Salas, 2014; Morales, 2010). Desde esta perspectiva, son una asignatura o campo de trabajo, de carácter interdisciplinario, cruzado por distintas ciencias, con un enfoque holístico, en el que se aprecia el diálogo de ciencias sociales, biofísicas y humanísticas, que desarrolla unas determinadas competencias personales y ciudadanas (National Council for Social Studies, 2010; Salas, 2014 y Marín, 2014), a partir de la resolución de problemas, ya sea de forma integrada o con base en la predominancia de una perspectiva histórica o geográfica" (p.16).

Por su parte, la Educación Cívica propone el “... mejoramiento de las capacidades necesarias para la vida colectiva” (MEP, 2009, p. 30). Pensando en esa formación como una necesidad que está presente en los sistemas democráticos y que debe llevar a la mejor toma de decisiones para la ciudadanía.

Ahora bien, la Escuela de Historia de la Universidad Nacional se encarga de la formación del profesorado e imparte la carrera como un combinado académico: Enseñanza de los Estudios Sociales y Educación Cívica. Como parte de las iniciativas de investigación y extensión de la carrera, es que nace la iniciativa del Proyecto Educación Continua Bimodal en el año 2016, con el objetivo de fortalecer los espacios de actualización del profesorado en este campo de trabajo.

De acuerdo con lo anterior, es necesario profundizar en el concepto de educación bimodal que en la actualidad tomó fuerza dentro de los contextos universitarios. Es así como diferentes programas de estudio se ajustan entre la presencialidad y la virtualidad como una forma de impulsar aprendizajes que se ajusten a las características de la comunidad aprendiente. Para efectos conceptuales, Araya (2013), citando a Heinze y Procter (2004), menciona que la educación bimodal "se desarrolla con la combinación efectiva de diferentes formas de exposición, modelos de enseñanza y estilos de aprendizaje, si se basa en una comunicación trasparente entre todas las personas participantes en un curso" (p. 3). 
Con este tipo de enseñanza se procura que el estudiantado pueda fortalecer las habilidades propias del trabajo colaborativo y que realmente se genere un aprendizaje significativo. Por medio de plataformas digitales, el estudiantado autodirige su aprendizaje y puede concretar investigaciones que apoyan su formación académica, ya sea de forma individual o en conjunto. Con el Proyecto Educación Continua Bimodal, la Escuela de Historia se planteó el objetivo de apoyar la formación continua del profesorado de educación media, con cursos de distintas temáticas que han formado parte de este proyecto y han capacitado a profesores de todo el territorio costarricense.

De igual forma, el proyecto busca fortalecer el aprendizaje activo derivado de la propuesta bimodal. Por lo tanto, el profesorado participante entra en contacto con actividades de mediación centradas en el trabajo colaborativo y autodirigido. De acuerdo con Schwartz y Pollishuke (2014), "los que participan en el aprendizaje activo están experimentando, interactuando, reflexionando y comunicándose" (p. 19). Por lo tanto, desde esta actividad académica se intenta concretar prácticas reflexivas, con el objetivo de que el profesorado lleve a las aulas propuestas innovadoras y acordes con su contexto laboral. El objetivo del aprendizaje activo es que el estudiantado sea el protagonista de su proceso de aprendizaje, decidiendo cómo y cuándo quiere aprender; con la guía del profesorado que orienta y motiva al colectivo aprendiente (Sierra, 2013).

El proyecto y sus cursos se basan en la propuesta teórica de la educación continua, como una forma de potenciar el crecimiento académico del profesorado, que se traducirá en una mejora constante de su práctica docente. Vargas (2001), citado por Andrade, Nava y Valverde (2009), define la educación continua como:

"una concepción de la educación, como un proceso permanente a lo largo de la vida, que involucra a la persona de manera integral y que se relaciona con cualquier tipo de actividad productiva en el ser humano, como un elemento esencial en la marcha de la civilización" (p.58).

Es decir, este tipo de educación surge de las necesidades de actualización del profesorado que busca profundizar en temáticas o metodologías que necesita fortalecer. La educación continua busca que los participantes se adecúen a las diferentes ofertas de capacitación y sobre todo al manejo del tiempo, que es una de las limitaciones que se presenta en este tipo de educación. En términos de Andrade, et al. (2010), la educación continua:

"permite garantizar el éxito profesional de muchas personas y contribuye a reformar su iniciativa individual, especialización, versatilidad, capacidad para la toma de decisiones, la interacción con los demás, 
trabajo en equipo, tanto en lo profesional como en lo social y familiar" (p. $58)$.

De acuerdo con lo anterior, desde el Proyecto de Educación Continua Bimodal y desde la Escuela de Historia de la Universidad Nacional se procura que cada uno de los cursos se adapte a las necesidades de actualización del profesorado, y que sean mediados por docentes altamente capacitados, con el objetivo de que el colectivo participante pueda obtener experiencias de aprendizaje significativo. Además, se hace uso de las plataformas tecnológicas de la universidad habilitadas para la realización de cursos virtuales o bimodales, con el objetivo de que las capacitaciones alcancen diferentes contextos del país.

Metodológicamente, este artículo se presenta como una experiencia de extensión basada en los insumos del curso bimodal de actualización para docentes de Estudios Sociales de la Dirección Regional de Heredia, realizándose un análisis de la experiencia y su relación con las TIC y su rol en los procesos de enseñanza y aprendizaje de la Historia. El curso contó con 39 participantes, de los cuales 20 concluyeron el ciclo formativo.

El objetivo central es demostrar que los procesos de actualización del profesorado relacionados a la disciplina histórica pueden verse impulsados con el uso de la tecnología educativa, siendo una herramienta valiosa para mejorar los procesos de enseñanza-aprendizaje.

\section{Desarrollo de la experiencia}

Tal como se mencionó anteriormente, el curso se desarrolló con la participación de 39 docentes de la Dirección Regional de Educación de Heredia. La coordinación se realizó con la asesora regional de Estudios Sociales; en conjunto con la asesoría se seleccionaron los temas y se efectuó la convocatoria respectiva para garantizar la asistencia del profesorado a las sesiones presenciales.

El curso bimodal tuvo una duración de 40 horas -20 horas presenciales y 20 horas virtuales-. Se inició el 17 de julio y finalizó el 30 de noviembre de 2017. Se ejecutó una sesión presencial por mes -5 horas por sesión-, cuyos contenidos eran complementados con lecturas y actividades de mediación propuestas en las sesiones virtuales. El curso se asentó en el Aula Virtual de Educación Permanente de la Universidad Nacional. Las sesiones presenciales se desarrollaron por historiadores y especialistas en relaciones internacionales. La tabla 1 muestra las temáticas y facilitadores de cada una de las sesiones presenciales del curso:

\section{Tabla 1}


El curso de actualización para docentes de Estudios Sociales de la Dirección Regional de Educación de Heredia: una experiencia desde el Proyecto de Educación Continua Bimodal

Temas y facilitadores de las sesiones presenciales del curso de actualización
docente

\begin{tabular}{|c|c|c|}
\hline Sesión & Tema & Facilitador \\
\hline I & $\begin{array}{l}\text { Historia ambiental y su pertinencia para la } \\
\text { Enseñanza de los Estudios Sociales y Cívica. } \\
\text { Huella ecológica }\end{array}$ & $\begin{array}{l}\text { Dr. Wilson Picado Umaña, } \\
\text { MSc. Maximiliano López } \\
\text { López. Escuela r de } \\
\text { Historia }\end{array}$ \\
\hline II & $\begin{array}{l}\text { América Latina: revoluciones sociales en el } \\
\text { contexto de la Guerra Fría y las } \\
\text { repercusiones actuales sobre los países de la } \\
\text { región. }\end{array}$ & $\begin{array}{l}\text { Dr. José } \\
\text { Morales. } \\
\text { Mscuelio Sandí } \\
\text { Historia. }\end{array}$ \\
\hline II & Los refugiados ambientales en el siglo XXI. & $\begin{array}{l}\text { Msc. Patricia Badilla } \\
\text { Gómez, Máster Alejandra } \\
\text { Barquero Ruiz. Escuela de } \\
\text { Historia. }\end{array}$ \\
\hline IV & $\begin{array}{l}\text { El islam y sociedades cristianas y } \\
\text { musulmanas en la Edad Media. }\end{array}$ & $\begin{array}{l}\text { Dr. Armando Torres } \\
\text { Fauaz. Escuela de } \\
\text { Historia. }\end{array}$ \\
\hline V & $\begin{array}{l}\text { El rol geopolítico de China en el contexto de } \\
\text { una economía globalizada. }\end{array}$ & $\begin{array}{l}\text { MSc. Carlos Cascante } \\
\text { Segura. Escuela de } \\
\text { Relaciones } \\
\text { Internacionales. }\end{array}$ \\
\hline
\end{tabular}

Fuente: Elaboración propia (2018), Proyecto de Educación Continua Bimodal, Escuela de Historia.

En las sesiones virtuales se continuó con el análisis de temas desarrollados en las sesiones presenciales, se incluían lecturas, videos y actividades de mediación que contribuyeran a mejorar el proceso de enseñanza y aprendizaje. Se utilizaron técnicas como foros académicos, análisis de noticias y cine-foros. Los participantes debían participar en las actividades propuestas y realizar diversas actividades de mediación como parte de la evaluación del curso.

Para el desarrollo de las sesiones virtuales se utilizó el modelo de enseñanza y aprendizaje impulsado por Salmon (2004), el cual involucra los siguientes niveles:

Tabla 2

Modelo de enseñanza y aprendizaje planteado por Salmon (2004)

Revista Perspectivas: Estudios Sociales y Educación Cívica - No 19

Julio - Diciembre, 2019 • pp. 1-11 


\begin{tabular}{|c|c|c|}
\hline $\begin{array}{l}\text { Nivel I } \\
\text {-Acceso y motivación. } \\
\text { - El facilitador da la } \\
\text { bienvenida a los } \\
\text { estudiantes y comunica } \\
\text { los requerimientos del } \\
\text { sistema. } \\
\text { - El facilitador orienta a } \\
\text { los estudiantes en el uso } \\
\text { de usuario y contraseña } \\
\text { de acceso. } \\
\text { - Actividades de } \\
\text { motivación. } \\
\text { - El estudiante explora la } \\
\text { organización del curso. }\end{array}$ & $\begin{array}{l}\text { Nivel II } \\
\text { - Socialización en línea } \\
\text { - Abrir espacios de } \\
\text { comunicación. } \\
\text { - Interacción facilitador- } \\
\text { estudiantes. } \\
\text { - Implementar normas de } \\
\text { comunicación. } \\
\text { - Crear un ambiente de } \\
\text { confianza, respeto y } \\
\text { empatía. }\end{array}$ & $\begin{array}{l}\text { Nivel III } \\
\text { - Intercambio de } \\
\text { información. } \\
\text { - Desarrollar de } \\
\text { estrategias de mediación } \\
\text { que generen entusiasmo, } \\
\text { independencia y confianza } \\
\text { entorno a la búsqueda de } \\
\text { información e } \\
\text { investigación. } \\
\text { - Implementar actividades } \\
\text { de mediación que } \\
\text { propicien la interacción } \\
\text { entre los estudiantes. } \\
\text { - Facilitar materiales que } \\
\text { contribuyan a la } \\
\text { construcción del } \\
\text { conocimiento. }\end{array}$ \\
\hline
\end{tabular}




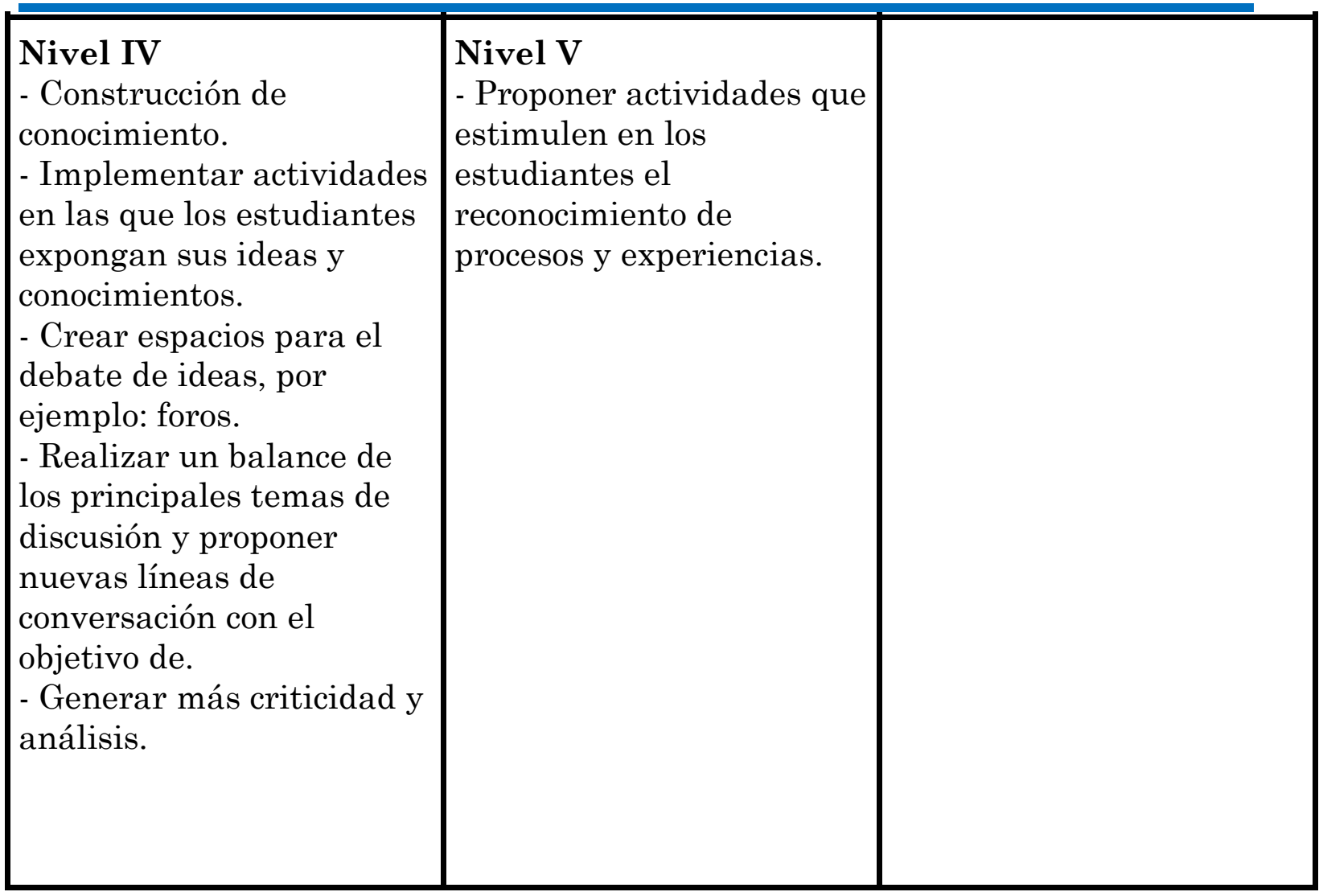

Fuente: Elaboración propia (2016), Proyecto de Educación Continua Bimodal, Escuela de Historia.

En la quinta sesión presencial desarrollada por el MSc. Carlos Cascante, se realizó una videoconferencia con estudiantes de la Escuela de Relaciones Internacionales que se encontraban en China, los cuales conversaron con los docentes sobre el rol geopolítico de China y las características socioculturales del país. Dialogo que fue muy enriquecedor para todos los presentes.

De los 39 participantes que matricularon el curso, 20 finalizaron el mismo. Se cree que la diferencia entre el número de inscritos y el número de participantes que finalizaron el curso se debe principalmente a los requerimientos de tiempo que conlleva el mismo, puesto que al ser cursos de aprovechamiento -40 horas- además de la asistencia a las sesiones presenciales se solicita el cumplimiento de tareas, ya que estos deben aprobarse con una nota igual o superior a 70. Cabe acotar que los cursos virtuales y bimodales constituyen un ahorro de dinero y tiempo, al evitarse desplazamientos constantes y gastos de traslado, de ahí que la demanda de estos dentro del Proyecto de Educación Continua sea grande.

Un aspecto importante que incluye el proyecto es que las actividades formativas se basan en necesidades reales de los docentes, los temas son actuales, atractivos y ofrecen el componente teórico y también el práctico, impulsando procesos de autorregulación del aprendizaje. Además, los 
participantes pueden desarrollar las actividades de acuerdo con su propio ritmo y estilo, puesto que estas son asincrónicas, lo que facilita su aprendizaje. Pueden trabajar en el lugar y tiempo que deseen.

Cabe resaltar que en este tipo de actividades los participantes tienen un papel más activo y son directamente responsables de su aprendizaje. El docente es un facilitador de procesos y de estrategias, un guía que invita a la reflexión partiendo de preguntas generadoras, de problemáticas, de experiencias cotidianas y de los conocimientos previos de los participantes, retomando el concepto de la formación del profesorado basado en prácticas reflexivas.

Para retroalimentar el proceso se evaluó el curso en conjunto con la asesora regional de Estudios Sociales, generándose los siguientes aspectos positivos: espacios de actualización sobre temas incluidos en los nuevos programas de estudio, con facilitadores de alto nivel la bimodalidad permitió a los docentes adecuar su tiempo a las exigencias del curso. En cuanto a los aspectos por mejorar, se mencionó el deseo de incluir más temas, capacitar sobre el uso de la plataforma y enviar el resultado de las evaluaciones con mayor rapidez. Aspectos que serán tomados en cuenta para futuros cursos.

\section{Principales conclusiones}

Los cursos bimodales constituyen una valiosa herramienta para la actualización de los docentes de secundaria, quienes, alternando sesiones presenciales y virtuales, logran adecuar su tiempo y espacio a procesos de capacitación necesarios para su desarrollo profesional. Por lo tanto, se consolidan como una propuesta de educación continua que el profesorado de Estudios Sociales y Educación Cívica busca para concretar espacios de formación.

La experiencia demostró que, en la actualidad, las tecnologías de la información y comunicación se convierten en herramientas necesarias para apoyar los procesos de educación continua. Esto quedó demostrado con las sesiones virtuales, donde el profesorado participó activamente y logró obtener aprendizajes significativos que pondrán en práctica con su estudiantado. De igual forma, las TIC desempeñan un papel importante en los procesos de enseñanza y aprendizaje de la Historia, dado que pueden ser utilizadas para implementar estrategias que permitan impulsar procesos de reflexión y análisis respecto a diversos tópicos.

Para mejorar los procesos de educación continua docente es necesario formular proyectos que conlleven la generación de redes de cooperación con diversas instituciones, siendo el Ministerio de Educación Pública esencial en este proceso. Por lo que las universidades no pueden trabajar aisladas, pues los docentes se desempeñan en un medio complejo que requiere un trabajo interinstitucional. Cabe recordar que la formación inicial de los educadores es responsabilidad de las universidades, las cuales también deben generar 
proyectos que coadyuven a la actualización de éstos. Entonces, si se desea mejorar la calidad de la educación se debe invertir en la formación docente.

\section{Referencias bibliográficas}

Andrade, J., Nava, M. y Valverde, J. (2009). La educación continua como proceso de formación académica en los alumnos egresados de las instituciones de educación superior en el estado de Sonora (México). Contabilidad y Negocios, 4(8), 57-62.

Araya, C. (2013). Diseño, ejecución y evaluación de un curso bimodal en la educación superior. Revista Electrónica Actualidades Investigativas en Educación, (7), 1-22.

Jiménez, I. y Vargas, C. (2011). Consideraciones para la implementación de un modelo de educación virtual: Revisión de áreas estratégicas. Revista Electrónica Educare, 15(2), 119-139.

Ministerio de Educación Pública (2009). Programa de Estudios de Educación Cívica. Tercer Ciclo de la Educación General Básica y Educación Diversificada. San José, Costa Rica: MEP.

Ministerio de Educación Pública (2016). Programa de Estudios Sociales para Tercer Ciclo de la Educación General Básica y Educación Diversificada y Técnica. San José, Costa Rica: MEP.

Presidencia de la República. (30 de mayo, 2016). MEP lanza nuevos programas de estudio en Ciencias y Estudios Sociales. Recuperado de https://presidencia.go.cr/comunicados/2016/05/mep-lanza-nuevosprogramas-de-estudio-en-ciencias-y-estudios-sociales/

Rodríguez, N., Espinoza, J. y Moreira, T. (2015). Cursos bimodales y presenciales: ¿Existen diferencias en los aprendizajes del estudiantado del TEC? Investiga TEC, (22), 3-4.

Schwartz, S. y Pollishuke, M. (2014). Aprendizaje activo. Una organización de la clase centrada en el alumnado (2da ed.). Madrid, España: NARCEA, S.A. DE EDICIONES.

Salmon, G. (2004). E-actividades. El factor clave para una formación en línea activa. Barcelona, España: Editorial UOC. 
Sierra, E. (2013). El aprendizaje activo como mejora de las actitudes de los estudiantes hacia el aprendizaje (tesis inédita de maestría). Universidad Pública de Navarra, Navarra, España. 